\title{
Self-organizing Ising model of financial markets
}

\section{Journal Article}

Author(s):

Zhou, W.-X.; Sornette, Didier

Publication date:

2007

Permanent link:

https://doi.org/10.3929/ethz-b-000002821

Rights / license:

In Copyright - Non-Commercial Use Permitted

Originally published in:

The European Physical Journal B 55(2), https://doi.org/10.1140/epjb/e2006-00391-6 


\title{
Self-organizing Ising model of financial markets
}

\author{
W.-X. Zhou ${ }^{1}$ and D. Sornette ${ }^{2, a}$ \\ 1 School of Business and Research Center of Systems Engineering, East China University of Science and Technology, \\ Shanghai 200237, P.R. China \\ 2 Department of Management, Technology and Economics, ETH Zurich, 8032 Zurich, Switzerland
}

Received 27 March 2006 / Received in final form 21 July 2006

Published online 25 October 2006 - (c) EDP Sciences, Società Italiana di Fisica, Springer-Verlag 2006

\begin{abstract}
We study a dynamical Ising-like model of agents' opinions (buy or sell) with learning, in which the coupling coefficients are re-assessed continuously in time according to how past external news (timevarying magnetic field) have explained realized market returns. By combining herding, the impact of external news and private information, we find that the stylized facts of financial markets are reproduced only when agents misattribute the success of news to predict return to herding effects, thereby providing positive feedbacks leading to the model functioning close to the Ising critical point.
\end{abstract}

PACS. 64.60.Ht Dynamic critical phenomena - 89.65.Gh Economics; econophysics, financial markets, business and management - 87.23.Ge Dynamics of social systems

\section{Introduction}

Social systems offer a fascinating field for the application of recent concepts and methods developed in Physics to tackle complex $N$-body systems with nonlinear feedbacks and many competing states. A long tradition started with the application of Ising models and its extensions to social interactions and organization [1-4]. A large set of economic models can be mapped to various versions of the Ising model to account for social influence in individual decisions (see [5] and references therein). Other recent works using the Ising model include models of bubbles and crashes $[6,7]$, a version with stochastic coupling coefficients which leads to volatility clustering and a power law distribution of returns at a single fixed time scale [8], and models of opinion polarization $[9,10]$. The dynamical updating rules of the Ising model can be shown to describe the formation of decisions of boundedly rational agents [11] or to result from optimizing agents whose utilities incorporate a social component [5]. The Ising model is one of the simplest models describing the competition between the ordering force of imitation or contagion and the disordering impact of private information or idiosyncratic noise, which leads already to the crucial concept of spontaneously symmetry breaking and phase transitions [12].

However, human beings are not spins, they can learn, that is, adapt the nature and strength of their interactions with others, based on past experience. In the lan-

\footnotetext{
a e-mail: dsornette@ethz.ch
}

guage of the Ising model, this amounts to generalizing to time-dependent coupling coefficients which reflect past experience. Here, we study a generalized Ising model of interacting agents buying and selling a single financial asset who base their decisions on a combination of imitation, external news and idiosyncratic judgments. The agents modulate their tendency to imitate based on their assessment of how past news have explained market returns. We distinguish between two possible updating rules that we refer to as (i) boundedly rational and (ii) irrational. We refrain from using the strict term "rational" which has a precise meaning in economics (subjective expectations that coincide with objective ones). Instead, we call "boundedly rational" those agents who decrease their propensity to imitate if news have been good predictors of returns in the recent past, as they correctly attribute the cause of the price moves. In the irrational version, agents misattribute the recent predictive power of news to their collective action, leading to positive self-reinforcement of imitation. We show that the model can reproduce the major stylized facts of financial markets only when the updating of the imitation strength is irrational, providing a direct test and the evidence for the importance of misjudgment of agents biased toward herding. This model also offers a dynamical derivation of the multifractal properties of the structure functions of the absolute values of returns and their consequences in the characteristic power law relaxations of the volatility after bursts of endogenous versus exogenous origins [13]. In a nutshell, our model suggests that 
the news serve as the substrate for fostering social interactions and reinforcing herding. Technically, the stylized facts result from the fact that the model operates around a critical point of an Ising model, with coupling coefficients which are time-dependent and endowed with a memory of past realizations, such that agents organize spontaneously within clusters of similar opinions, which become very susceptible to small external influences, such as a change of news. This may explain the occurrence of crashes [14].

\section{Presentation of the model}

Consider $n^{2}$ agents interacting within a $n \times n 2 \mathrm{D}$ square lattice network $\mathcal{N}$ (we have verified that the properties described below are not sensitive to $n$ in the range 20-100 that we tested). At each time step $t$, agent $i$ places a buy $\left(s_{i}(t)=+1\right)$ or sell $\left(s_{i}(t)=-1\right)$ order according to the following process

$$
s_{i}(t)=\operatorname{sign}\left[\sum_{j \in \mathcal{N}} K_{i j}(t) E_{i}\left[s_{j}\right](t)+\sigma_{i} G(t)+\epsilon_{i}(t)\right],
$$

where $E_{i}\left[s_{j}\right](t)$ is the expectation formed by agent $i$ on what will be the decision of agent $j$ at the same time $t$. An agent $i$ imitates only her friends, that is $K_{i j}=0$ if $i=j$ or $j$ is not a friend of (connected to) $i$.

Expression (1) reduces to the dynamical stochastic formulation of the Ising model, when replacing the expectation $E_{i}\left[s_{j}\right](t)$ by $s_{j}$ : then, the first term derives from the gradient of the Ising Hamiltonian with respect to the spin value, the second term $\sigma_{i} G(t)$ plays the role of an external magnetic field and the third term embodies the thermal fluctuations. Note that the dynamical stochastic formulation of the Ising model is equivalent for its statistical properties to the more standard form obtained from the partition function calculated with an Hamiltonian written in terms of a sum over pairs of spins of the form $-\sum_{i, j} K_{i j} s_{i} s_{j}-G \sum_{i} s_{i}$, where the pairs are connected according to some graph topology reflecting the physics or social nature of the problem. The stochastic dynamical definition of the Ising model is obtained by writing that a given spin is updated proportionally to the derivative of the Hamiltonian with respect to this spin. This so-called over-damped dynamics leads to our above equation (1). The use of Ising models of a similar type to describe opinion formation has a long history [15-18]. The present work constitutes an extension to study non-variational dynamics by allowing non symmetric couplings evolving and adapting in time. The impact of external news that we study here is reminiscent of Sethna et al. [19] who found a transition in a random field Ising model as a function of a changing external field. Our work differs however in several ways: local spins are not pinned by local random fields and our external magnetic field is stochastic. In addition, agents (spins) evolve their coupling by adapting as a function of the past behavior of the global systems in response to the stochastic external news.

From the view point of a model of interacting investors, expression (1) embodies three contributions:
1. imitation in which $K_{i j}$ is the relative propensity of the trader $i$ to be contaminated by the sentiment of her friend $j$;

2 . the impact $G(t)$ of external news (positive resp. negative for favorable resp. unfavorable news), which is an i.i.d. standard Gaussian noise, and $\sigma_{i}$ is the relative sensitivity of agent's sentiment to the news, uniformly distributed in the interval $\left(0, \sigma_{\max }\right)$ and frozen to represent the heterogeneity of the agents;

3 . the idiosyncratic judgment $\epsilon_{i}(t)$ associated with private information, assumed to be normally distributed around zero with an agent-dependent standard deviation $s_{\epsilon, i}$ equal to the sum of a common constant $C V$ and of a uniform random variable in the interval $[0,0.1]$ again to capture the heterogeneity of agents.

We have tested several implementation of the formation of expectations $E_{i}\left[s_{j}\right](t)$ in (1), such as backward looking $\left(E_{i}\left[s_{j}\right](t)=s_{j}(t-1)\right.$ for all $i$ and $j$ 's) or information cascades along specific chains within the network [20], which give similar results.

Our model makes the simplifying approximation that the agents' decisions are dominated by their interactions with other agents and with external news as well as the effect of their idiosyncratic analysis. We neglect the possible influence of the price itself on the decision making process. This assumption is convenient to simplify the analysis and makes more transparent the relation with the Ising model. Our model is thus at the opposite end to the class of agent-based models, such as the minority and majority games, which emphasize the oppositive view point that agents do not interact directly but all look at the price aggregating their decisions. While neither assumption is realistic, it is interesting to study their impact separately. The present paper offers such an attempt. At first sight, neglecting the possible influence of the price itself on the decision making process may appear unrealistic. But, this is actually the strategy adapted by socalled "value"-investors who analyze the theoretical value of a firm based on fundamental economic indicators and allocate correspondingly their portfolio basically independently of the stock market price. Indeed, in the view of value investors, the erratic variations of the instantaneous stock market price are perceived as noisy stochastic perturbations which are irrelevant to a sound medium- and long-term investment process. Our neglect of the possible influence of the price itself on the decision making process thus amounts to considering mostly a crowd of valueinvestors and to study their decision process, assuming either that they take a boundedly rational or an irrational stance with respect to external news.

The market price is updated according to $p(t)=p(t-1) \exp [r(t)]$ where the return $r(t)$ obeys

$$
r(t)=\frac{1}{\lambda N} \sum_{i \in \mathcal{N}} s_{i}(t),
$$

where $\lambda$ measures the market depth or liquidity. The return is thus proportional to the "magnetization" or aggregated decisions of the agents. Note that agents do 
not use the market returns to form their expectations and to make decisions, except indirectly through the following term. We account for the adaptive nature of agents and their learning abilities by updating the coefficient of influence of agent $j$ on agent $i$ according to the following rule:

$$
K_{i j}(t)=K_{i}(t)=b_{i}+\alpha K_{i}(t-1)+\beta r(t-1) G(t-1) .
$$

The idiosyncratic imitation tendency $b_{i}$ of agent $i$ is uniformly distributed in $\left(0, b_{\max }\right)$ and frozen. The coefficient $\alpha$ quantifies the persistence of past influences on the present. It captures the fact that social connections evolve slowly and exhibit significant persistence, as documented in numerous studies $[21,22]$. Networks of investors communicating their opinions and sentiment on the stock market are similarly persistent. The last term with $\beta \neq 0$ quantifies how agent $i$ updates her propensity for imitation based on the role of the exogenous news $G(t)$ in determining the sign and amplitude of the observed return in the preceding time period. Note that, by construction, the model is non-variational as $K_{j i}(t) \neq K_{i j}(t)$ in general if the $b_{i}$ 's are different, but this is not crucial for our results. This means that the dynamics can not be derived from a Hamiltonian (or energy functional). A famous example of non-variational dynamics is turbulent hydrodynamics.

The case $\alpha=0$ was previously investigated in part by Gonçalves in Ref. [20]), who provided a first generalization step of the model of imitative noise traders introduced in [6]. The present work generalizes Gonçalves's model further by introducing both a memory of the coefficient of influence $(\alpha \neq 0)$ and the possibility of nonrational behavior $(\beta>0)$. The memory term captures the fact that agents do not change their propensity to imitate completely upon receipt of a new information, but rather combine this new information with their past experience. Considering the possibility that $\beta$ can be positive addresses a fundamental issue, which is the possibility that investors misinterpret information, which is here the real source of predictability. This addresses a large concern of the literature on behavioral finance, namely how mutually-reinforcing optimism may lead to price distortion and anomalies.

The sign of the coefficient $\beta$ is crucial. For $\beta<0$, agent $i$ is less and less influenced by other agents, the better has been the success of the news in determining the direction and amplitude of the market return. This process is self-reinforcing since, as $K_{i j}$ decreases, the dominant term becomes $\sigma_{i} G(t)$, which further ensures that the news correctly predict the decision of agents and therefore the direction of the market move, thus decreasing further the coefficient of influence $K_{i j}$. Reciprocally, agents tends to be more influenced by others when the news seems to incorrectly predict the direction of the market. The news being not reliable, the agents turn to other agents, believing that others may have useful information. This is in agreement with standard economics which views the stock market as an efficient machine transforming all news into prices.

For $\beta>0$, the more the news predict the direction of the market, the more the agents imitate other agents. This

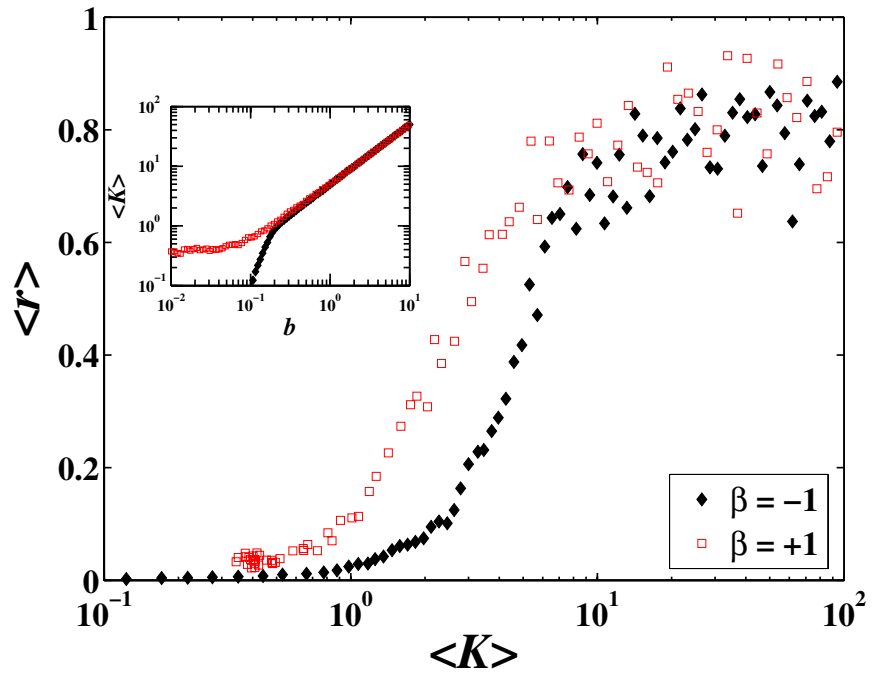

Fig. 1. (Color online) Average return $\langle r\rangle$ over time as a function of the average coefficient of influence $\langle K\rangle$ over time, where $K$ is defined in (3), obtained by varying $b_{\max }$ from 0.1 to 100 . Inset: dependence of $\langle K\rangle$ as a function of $b_{\max }$.

is the "irrational" case where agents either mis-attribute the origin of the market moves to herding rather than to the impact of news, or misinterpret the exogenous character of news in terms of endogenous herding or infer that other agents will be following more eagerly as a group the direction given by the news. This may occur due to mutually-reinforcing optimism [23] and overconfidence [24]. This case captures the evidence from a growing literature in neuro-psychology and behavioral finance documenting that feelings, mood and overconfidence affects perception through misattribution leading to incorrect judgments or affecting people's ability to process information $[25-31]$.

We fix $\lambda=40$ in our simulations to obtain returns with amplitudes comparable to that of empirical observations and show results for $\alpha=0.2$. Similar results are obtained for $\alpha=0.4,0.6$, and 0.8 . We have explored the properties of the model in the parameter space of $b_{\max }, \sigma_{\max }$ and $C V$. There is no loss of generality in fixing $|\beta|=1$ to explore the relative importance of the term $\beta r(t-1) G(t-$ 1 ), since the typical scale of the $K_{i}$ 's is set by $b_{\max }$ whose amplitude is varied in our numerical exploration.

\section{Results}

Figure 1 illustrates the existence of an Ising-like phase transition, as a function of the control parameter $b_{\max }$ for both regimes $\beta= \pm 1$, visualized by the S-shape dependence of the average return $\langle r\rangle$ over time as a function of the average coefficient of influence $\langle K\rangle$ over time. The abruptness of the jump in $r$ is controlled by the size of the system (here $50 \times 50$ agents) as in standard finite-size effects. As we describe below, we find realistic properties for $b_{\max }$ in the range $0.2-0.6$ which correspond precisely to range of $\langle K\rangle$ over which the transition occurs. 


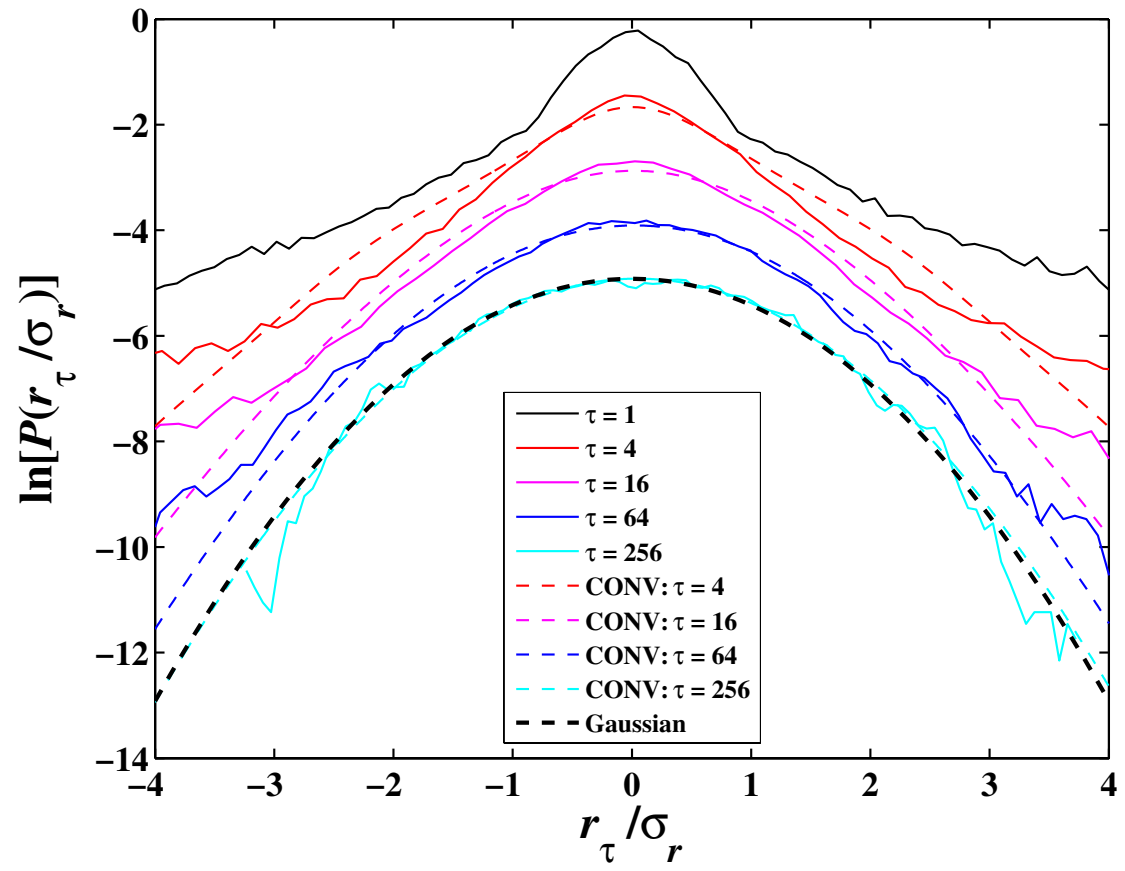

Fig. 2. (Color online) Probability distribution density (in logarithmic scales) of logreturns at different time scales $\tau$ of a price time series obtained with $\lambda=40, \alpha=0.2$, $b_{\max }=0.3, \sigma_{\max }=0.03$, and $C V=0.1$. The $\log$-returns $r_{\tau}$ are normalized by their corresponding standard deviations $\sigma_{\tau}$. The pdf curves are translated vertically for clarity. The thick dashed line is the Gaussian pdf. The other dashed lines have been obtained by $\tau$-fold convolutions of the pdf of the onetime step return $r_{1}(t)=\ln p(t) / p(t-1)$.
We study relatively small systems (from $20 \times 20$ to $100 \times 100)$ because there are many evidences that the effective number of investors moving the price of a given asset at any time is of the order of tens to a hundred, no more (see for instance [32]). Considering 400 to 10000 agents thus seems sufficient for our purpose.

\subsection{Case $\beta=-1$ ("boundedly rational" agents)}

For $\beta=-1$, and $b_{\max }<0.6$, the attractor of the dynamics is characterized by negligible imitation and only the news and private information terms are important for the dynamics, due to the self-reinforcing mechanisms discussed above leading to the system functioning in the "paramagnetic" phase. The collective decision $\sum_{i} s_{i}$ and therefore the market return have little or no relationship with the external news. Hence, the term $\beta r(t+1) G(t)$ takes random signs from one time step to the next, leading to an effective random forcing added to the autoregressive equation $K_{i}(t)=b_{i}+\alpha K_{i}(t-1)$. We thus expect Gaussian distributions of returns when $b_{i} /(1-\alpha)$ is smaller than $K_{c}$ and bimodal distributions when $b_{i} /(1-\alpha)>K_{c}$ reflecting the slaving of the global opinion to the sign of the news. Our simulations, which have scanned 480 different models for $b_{\max }$ from 0.1 to 0.5 with spacing $0.1, \sigma_{\max }$ from 0.005 to 0.08 with spacing 0.005 , and $C V$ from 0.1 to 1.1 with spacing 0.2 , confirm this prediction. Consider the distribution of returns $r_{\tau}(t)=\ln [p(t) / p(t-\tau)]$ at different time scales $\tau$. For large idiosyncratic noise (large $C V$ ) and not too large $b_{\max }$, the distribution of returns is Gaussian for all time scales $\tau$. For smaller $C V$ 's and larger $b_{\max }$, we observe multimodal return distributions. In the parameter space that we have explored and notwithstanding our best attempts, we have not been able to find a set of parameters leading to distributions of returns exhibiting a monomodal shape with fat tails for small time scales, evolving slowly towards Gaussian distributions at large time scales, as can be observed in empirical data [33-36]. In addition, the correlation function of returns at time scale $\tau\left(C_{\tau}(r, r)(l) \equiv\left\langle r_{\tau}(t) r_{\tau}(t+l)\right\rangle\right.$ where $l$ is the lag) and of volatilities $\left(C_{\tau}(|r|,|r|) \equiv\left\langle\left|r_{\tau}(t)\right|\left|r_{\tau}(t+l)\right|\right\rangle\right)$ have similar amplitudes and decay with the same characteristic time scale as a function of time lag. This is very different from the observed correlations of financial markets, with very short memory for returns and long-memory for the volatility.

We emphasize that there are no significant correlations of returns for this "boundedly rational" case. The main point is that the correlations of the returns and of the volatility are similar, with the same characteristic time scale of decay, in contradiction with real data for which the correlation time scale of returns is of the order of minutes while the time scale of the correlation of volatility is days or weeks. These observations do not imply arbitrage opportunities for boundedly rational agents. Indeed, it is well-known that a small correlation time for returns does exist in real market but does not lead to arbitrage opportunities due to market friction (transaction costs and slipage).

\subsection{Case $\beta=+1$ ("mis-attributing" agents)}

For $\beta=1$, we obtained the following main stylized facts of financial stock markets: (i) distributions of returns at different time scales $\tau$ (Fig. 2); (ii) correlation function of returns and of the absolute value of the returns (Fig. 3); (iii) scaling of the moments of increasing orders of the absolute values of the returns (qualifying multifractality); (iv) the existence of a hierarchy of exponents controlling the relaxation of the volatility after an endogenous shock, another 


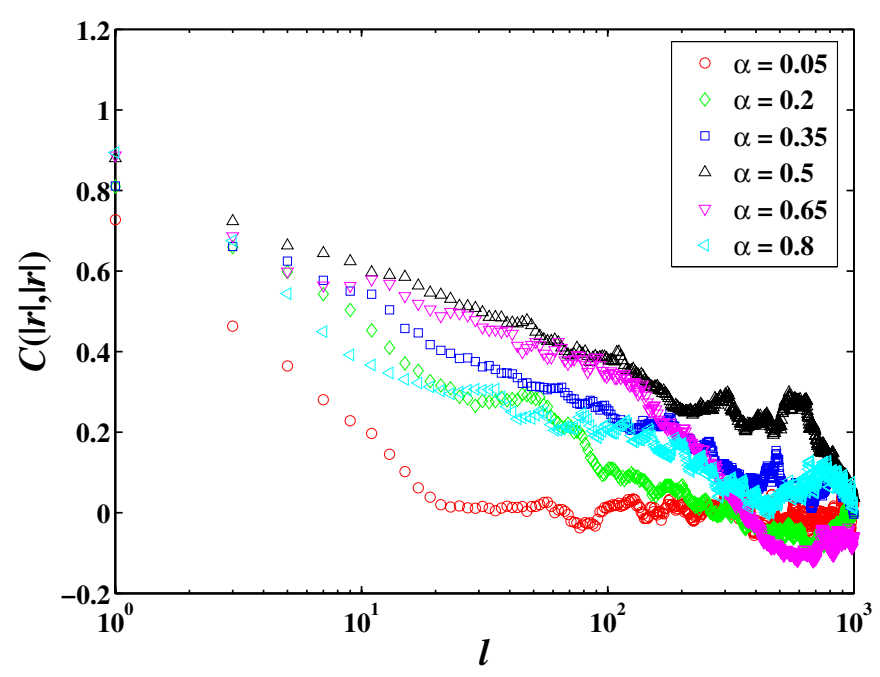

Fig. 3. (Color online) Impact of $\alpha$ on the auto-correlation of the absolute values of the returns. A linear dependence $C(l)=$ $1-k \ln (l / T)$ in the logarithm of the time lag $l$ shown here is undistinguishable from a power law $C(l) \sim 1 /(l / T)^{k}$ for $k=0.1-0.3$ over these three orders of magnitude, similarly to empirical results. Here, $T$ is a constant, which takes the meaning of the "integral" scale in the MRW model.

hallmark of multifractality (Fig. 4); (v) the existence of bubbles and crashes (see [37] for a detailed description). We have explored 160 models with $b_{\max }$ from 0.1 to 0.5 with spacing $0.1, \sigma_{\max }$ from 0.01 to 0.08 with spacing 0.01 , and $C V$ from 0.1 to 0.7 with spacing 0.2 . For each model, we generate time series of length equal to $10^{5}$ time steps. We have found a large set of parameter combinations which lead to realistic stylized facts. The results presented here are for $\left(b_{\max }=0.3, \sigma_{\max }=0.03, C V=0.1\right)$ which is typical.

Figure 2 shows the evolution of the pdf's of returns from stretched exponential or power laws at short time scales that cross over smoothly to a Gaussian law at the largest shown time scale, in excellent agreement with empirical facts [33-36]. Note the difference between the continuous and dashed lines for $\tau=4,16$, and 64 , which expresses the existence of significant dependence in the time series of returns. Such behavior is very similar to what is observed in real data.

The temporal correlation of the $\log$ returns $r_{1}$ as a function of the time lag $\ell$ exhibits a very short correlation time, of duration smaller than one time step (not shown). In contrast, the temporal correlation of the absolute value of $\log$ returns $r_{1}$ ("volatility"), taken as a proxy for the volatility, exhibits long-range dependence up to approximately 1000 time steps. The linear-log relationship suggested by the plots in Figure 3 are predicted by the multifractal random walk (MRW) [38], which provides an excellent model of many properties of financial time series [39]. The MRW depends only on three parameters: the multifractal parameter $\lambda^{2} \approx 0.02-0.04$, the integral time scale $T \approx 1$ year and the standard deviation of returns. Com-

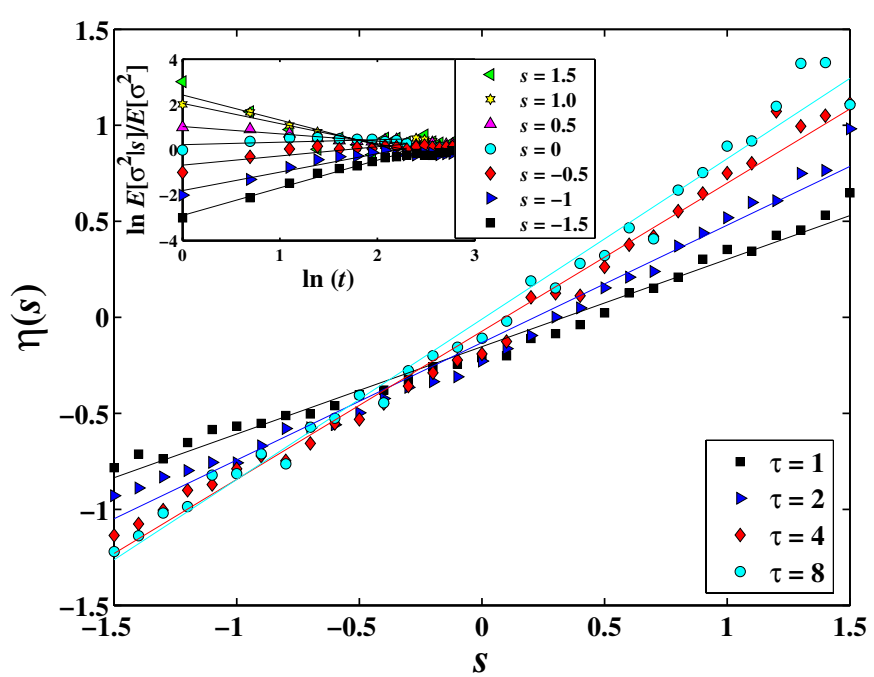

Fig. 4. (Color online) For a given relative log-amplitude $s$ of a local burst of volatility occurring at some time $t_{s}$, we translate and superimpose all time series starting at those times of local bursts of the same amplitude $s$. Averaging over these time series of volatility obtains the average conditional relaxation function of the volatility $E\left[|r|^{2}(t) \mid s\right] \sim t^{-\eta(s)}$ following a local burst of volatility of amplitude $\propto e^{s}$. The prediction (9) gives the straight lines for $T=200$. The inset shows the average normalized conditional volatility $E\left[|r|^{2} \mid s\right] / E\left[|r|^{2}\right]$ as a function of the time after the local burst of volatility for different logamplitudes $s$. The figure shows the exponents $\eta(s)$ measured as the slopes of the curves in the inset for $\tau=1,2,4$, and 8 .

paring the dependence properties of the returns and of the volatility suggests that one trading day corresponds roughly to 5 time steps of the model. This correspondence translates into a integral time scale $T$ of about 200 days, which is compatible with empirical estimates for the MRW [40]. The MRW also predicts (and this is well-verified by empirical data) that the autocorrelation functions of $\left|r_{\tau}(t)\right|$ for different $\tau$ should superimpose for time lags larger than their respective $\tau$ [38]. This prediction is also approximately observed in our model (not shown).

One of the stylized facts is the long-memory of volatility, which is usually characterized by a decay of the volatility autocorrelation $C(l)$ as a power-law with a very small exponent $k$ (around $0.2-0.3$ ). In contrast, Figure 3 shows that $C(l)$ is well-approximated by

$$
C(l)=1-k \ln (l / T) .
$$

This expression is actually undistinguishable from the often quoted power law

$$
C(l) \sim 1 /(l / T)^{k}
$$

if the exponent $k$ is typically smaller than 0.3 , for the range of time lags $l$ usually available. Indeed, expression (5) can 
be written as

$$
\begin{aligned}
C(l) \sim 1 /(l / T)^{k} & =\exp [-k \ln (l / T)] \\
& =1-k \ln (l / T)+(1 / 2)(k \ln (l / T))^{2}+\ldots
\end{aligned}
$$

by expanding the exponential. Thus, for $k \ln (l / T)<1$, expressions (4) and (5) are approximately the same and, in general, the financial data does not allow one to distinguish between the two formulations.

Another important stylized facts is the multifractal structure of the absolute values of log-returns [41,40]. We verify the existence of a strong multifractality in our time series (not shown) expressed by the scaling of the structure function

$$
M_{q}(\tau) \equiv\left\langle\left|r_{\tau}\right|^{q}\right\rangle \sim \tau^{\xi_{q}}
$$

with exponents $\xi_{q}$ exhibiting a clear nonlinear dependence as a function of the order $q$ of the structure function. Rather than showing this standard looking multifractal spectrum, Figure 4 presents another striking signature of multifractality discovered first in empirical data [13]: the MRW predicts a continuous spectrum of exponents $\eta(s)$ for the relaxation of the volatility

$$
E\left[|r|^{2}(t) \mid s\right] \sim t^{-\eta(s)}
$$

from a local peak as a function of its amplitude $\propto e^{s}$ given by $[13]$

$$
\eta(s)=\frac{2 s}{3 / 2+\ln (T / \tau)} .
$$

Relation (9) together with (8) describes a continuous spectrum of exponents, that is, a continuum of singularities in the time domain. For the MRW, the two statements can be seen to be equivalent. More generally, multifractality in the time domain has two equivalent signatures: (i) a continuous spectrum of exponent $\xi_{q}$ for the moments (also called structure functions) of order $q$ and (ii) a continuous set of Hölder singularities (or local fractal singularities). The exponents $\eta(s)$ correspond to the set of Hölder exponents quantifying the local Hölder singularities in the time domain, which provide a diagnostic of the multifractal measure equivalent to the moment exponents $\xi_{q}$.

\section{Concluding remarks}

We have presented a model which allows us to test within the same framework the comparative explanatory power of boundedly rational versus irrational agents, with respect to the main stylized facts of financial markets. The assumptions of our model are quite standard, taken one by one. What we have done is to put them together to obtain a single model in which the question on whether boundedly rational agents or irrational agents better explain the observed regularities of the financial markets can be investigated. In particular, the "irrational" regime $(\beta=+1)$ derives from the evidence from a growing literature in neuro-psychology and behavioral finance documenting that feelings, mood and overconfidence affects perception through misattribution leading to incorrect judgments or affecting people's ability to process information. Misattribution is a fundamental and ubiquitous psychological trait of humans and its impact on trading is now recognized and the recognition of its importance is growing. One contribution of our work is to suggest an observation consequence of the misattribution trait shared by humans.

The model studied here asks directly the question whether the agents aggregate collectively in a boundedly rational or irrational behavior. Galam [17] has introduced irrationality in a different way via a "temperature" introducing a stochastic element in the decision process modeled within a random field Ising model. In contrast, our model has allowed us to falsify the question on the bounded rationality versus irrationality of investors, through a simple implementation emphasizing the importance of biases misrepresenting the news, rather than the role of stochastic errors.

We are grateful to Gonçalves for useful discussions. This work was partially supported by the National Natural Science Foundation of China (Grant No. 70501011), the Fok Ying Tong Education Foundation (Grant No. 101086), and the Shanghai Rising-Star Program (Grant No. 06QA14015).

\section{References}

1. E. Callen, D. Shapero, Phys. Today 27, 23 (1974)

2. E.W. Montroll, W.W. Badger, Introduction to Quantitative Aspects of Social Phenomena (Gordon and Breach, New York, 1974)

3. S. Galam, Y. Gefen, Y. Shapir, Math. J. Soc. 91, (1982)

4. A. Orléan, J. Econ. Behav. Org. 28, 274 (1995)

5. D. Phan, M.B. Gordon, J.-P. Nadal, in Cognitive Economics - An Interdisciplinary Approach, edited by P. Bourgine, J.-P. Nadal (Springer, 2004), pp. 335-354

6. A. Johansen, O. Ledoit, D. Sornette, Int. J. Theoret. Appl. Fin. 3, 219 (2000)

7. T. Kaizoji, S. Bornholdt, Y. Fujiwara, Physica A 316, 441 (2002)

8. A. Krawiecki, J.A. Holyst, D. Helbing, Phys. Rev. Lett. 89, 158701 (2002)

9. S. Galam, Physica A 336, 49 (2004)

10. D. Stauffer, in Modeling Cooperative Behavior In The Social Sciences, edited by J. Marro, P.L. Garrido, M.A. Muñoz, AIP Conference Proceedings, Granada, Spain, (2005), Vol. 779, pp. 56-68

11. B.M. Roehner, D. Sornette, Eur. Phys. J. B 16, 729 (2000)

12. B.M. McCoy, T.T. Wu, The Two-Dimensional Ising Model (Harvard University Press, Cambridge, Mass, 1973)

13. D. Sornette, Y. Malevergne, J.-F. Muzy, Risk 16, 67 (2003)

14. D. Sornette, Why Stock Markets Crash: Critical Events in Complex Financial Systems (Princeton University Press, Princeton, 2003)

15. S. Galam, S. Moscovici, Eur. J. Soc. Psychology 21, 49 (1991) 
16. S. Galam, in Understanding Group Behavior: Consensual Action by Small Groups, edited by E.H. Witte, J.H. Davis (Lawrence Erlbaum Associates, New Jersey, 1996), Vol. I, Chap. 12, pp. 293-312

17. S. Galam, Physica A 238, 66 (1997)

18. Q. Michard, J.-P. Bouchaud, Eur. Phys. J. B 47, 151 (2005)

19. J.P. Sethna, K. Dahmen, S. Kartha, J.A. Krumhansl, B.W. Roberts, J.D. Shore, Phys. Rev. Lett. 70, 3347 (1993)

20. C.P. Gonçalves, Artificial financial market model, unpublished (2003)

21. J.J. Suitor, B. Wellman, D.L. Morgan, Soc. Networks 19, 1 (1997)

22. B. Wellman, R.Y.-L. Wong, D. Tindall, N. Nazer, Soc. Networks 1, 27 (1997)

23. C. Heath, R. Gonzalez, Organizational Behavior \& Human Decision Processes 61, 305 (1995)

24. P.R. Darke, J.L. Freedman, Personality \& Social Psychology Bulletin 23, 378 (1997)

25. N. Schwarz, G.L. Clore, J. Person. Soc. Psych. 45, 513 (1983)

26. N. Frijda, Cognition and Emotion 1, 235 (1988)

27. Handbook of Motivation and Cognition: Foundations of Social Behavior, edited by R.M. Sorrentino, E.T. Higgins, Vol 2. (Guilford Press, New York, 1990)
28. K. Savitsky, V.H. Medvec, A.E. Charlton, T. Gilovich, Personality \& Social Psychology Bulletin 24, 529 (1998)

29. R.A. Weber, Y. Rottenstreich, C. Camerer, M. Knez, Org. Sci. 12, $582(2001)$

30. B.M. Lucey, M. Dowling, J. Econ. Surveys 19, 211 (2005)

31. J.R. Nofsinger, J. Behav. Fin. 6, 144 (2005)

32. P. Coyne, J.W. Witter, The McKinsey Quarterly, Number $2,(2002)$

33. S. Ghashghaie, W. Breymann, J. Peinke, P. Talkner, Y. Dodge, Nature 381, 767 (1996)

34. R.N. Mantegna, H.E. Stanley, An Introduction to Econophysics: Correlations and Complexity in Finance (Cambridge University Press, Cambridge, 2000)

35. Y. Malevergne, V. Pisarenko, D. Sornette, Quant. Finance 5, 379 (2005)

36. Y. Malevergne, V. Pisarenko, D. Sornette, Appl. Fin. Econ. 16, 271 (2006)

37. D. Sornette, W.-X. Zhou, Physica A 370, 704 (2006)

38. E. Bacry, J. Delour, J.-F. Muzy, Phys. Rev. E 64, 026103 (2001)

39. T. Lux, Quant. Finance 1, 632 (2001)

40. J.-F. Muzy, D. Sornette, J. Delour, A. Arnéodo, Quant. Finance 1, 131 (2001)

41. B.B. Mandelbrot, Fractals and Scaling in Finance (Springer, New York, 1997) 